(c) 2020 Universidad Nacional Autónoma de México, Facultad de Estudios Superiores Zaragoza.

Este es un artículo Open Access bajo la licencia CC BY-NC-ND (http://creativecommons.org/licenses/by-nc-nd/4.0/).

TIP Revista Especializada en Ciencias Químico-Biológicas, 23: 1-10, 2020.

https://doi.org/10.22201/fesz.23958723e.2020.0.233

\title{
Caracterización e identificación de compuestos bioactivos con actividad antioxidante de la cáscara, pulpa y semilla del fruto de tejocote (Crataegus mexicana)
}

\author{
M. Viviana Robles-Botero ${ }^{1}$, Elba Ronquillo-de Jesús ${ }^{2}$, \\ Cinthya N. Quiroz-Reyes ${ }^{1}$ y Miguel A. Aguilar-Méndez ${ }^{1 *}$ \\ ${ }^{1}$ Instituto Politécnico Nacional, Centro de Investigación en Ciencia Aplicada y \\ Tecnología Avanzada. Legaria \# 694, Col. Irrigación, Ciudad de México, 11500, México. \\ ${ }^{2}$ Universidad Politécnica de Francisco I. Madero, Dirección de Ingeniería \\ Agroindustrial, Tepatepec, Hidalgo, 42660, México. E-mail: *maguilarme@ipn.mx
}

\begin{abstract}
RESUMEN
El tejocote (Crataegus mexicana) es una planta nativa de México. Su fruto no sólo es utilizado como alimento para el consumo humano, sino también como ornamento en celebraciones y en la medicina tradicional. A pesar de esto, se le considera un cultivo subutilizado y su aporte de compuestos con actividad antioxidante ha sido poco estudiado. El objetivo de este trabajo fue caracterizar e identificar metabolitos secundarios extraídos de las fracciones del fruto para determinar su potencial antioxidante: (1) cáscara, (2) pulpa y (3) semilla. Se cuantificó el contenido de fenoles y flavonoides totales, flavan3-ol y proantocianidinas. Además, la actividad antioxidante de las tres fracciones fue cuantificada mediante dos métodos: (1) reducción del hierro (FRAP) e (2) inhibición del radical libre 2,2-difenil-1-picrilhidracilo (DPPH). Se identificaron los principales compuestos presentes en cada una de las fracciones a través de cromatografía de líquidos de alta resolución (HPLC). Los resultados indicaron que fue en el extracto de la semilla donde se presentó el mayor contenido de fenoles y flavonoides totales, así como el mejor potencial antioxidante, el cual estuvo directamente relacionado con el contenido de compuestos extraídos. En las tres fracciones del fruto se registró la presencia de epicatequina, quercetina 3-D-galactósido y ácido ascórbico. Además, en semilla se identificó la catequina y vitexina, mientras que en cáscara y pulpa se observó la presencia de ácido clorogénico y procianidina B2.

Palabras clave: Crataegus mexicana, antioxidante, polifenol, extracción asistida por ultrasonido.
\end{abstract}

Characterization and identification of bioactive compounds with antioxidant activity from peel, pulp and seed of tejocote fruit (Crataegus mexicana)

\begin{abstract}
Tejocote (Crataegus mexicana) is a native plant from Mexico. Its fruit is not only used as food for human consumption but also as an ornament in celebrations and traditional medicine. Despite this, it is considered an underutilized crop, and its antioxidant-containing compounds have only been minimally studied. The objective of this work was to characterize and identify secondary metabolites extracted from three fruit fractions to determine their antioxidant potential: (1) peel, (2) pulp and (3) seed. Phenols and total flavonoid, flavan-3-ol, and proanthocyanidins contents were determined. Also, antioxidant activities of the three fractions were quantified by two methods: (1) iron reduction (FRAP) and (2) inhibition of the 2,2-diphenyl-1- picrylhydrazyl free radical (DPPH). The main compounds present in each of the fractions were identified through high-performance liquid chromatography (HPLC). The results showed that the seed extract exhibited the highest phenol and total flavonoid contents and the best antioxidant potential, which was directly related to the content of extracted compounds. The presence of epicatechin, quercetin 3-D-galactoside, and ascorbic acid was recorded in the three fractions of the fruit. In addition, catechin and vitexin were identified in seed, while in the peel and pulp the presence of chlorogenic acid and procyanidin B2 was detected.
\end{abstract}

Keywords: Crataegus mexicana, antioxidant, polyphenol, ultrasound-assisted extraction.

Artículo recibido el 20 de noviembre del 2019.

Artículo aceptado el 23 de junio del 2020. 


\section{INTRODUCCIÓN}

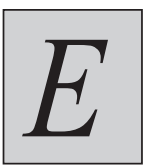

1 género Crataegus agrupa alrededor de 280 especies distribuidas en el hemisferio Norte (Lozano-Grande, Valle-Guadarrama, Aguirre-Mandujano, LobatoCalleros \& Huelitl-Palacios, 2016), 13 de ellas son originarias de México (Phipps, 1997). Las especies del género Crataegus también se encuentran en Asia, Europa y América del Norte (Edwards, Brown, Talent, Dickinson \& Shipley, 2012). En flores, hojas, raíz y fruto del género Crataegus se han identificado flavonoides, ácidos fenólicos, azúcares, terpenos y otros compuestos (Edwards et al., 2012; García-Mateos, Aguilar-Santelises, Soto-Hernández, Nieto-Ángel \& Kite, 2012). Algunas variedades de este género se describieron en farmacopeas antiguas y actualmente se emplean como alternativa para el tratamiento de padecimientos relacionados con el sistema cardiovasculary las vías respiratorias (Rigelsky \& Sweet, 2002). Sin embargo, su empleo en la medicina tradicional mexicana es bajo, si se compara con el que se presenta en otras regiones del mundo (Cervantes-Paz et al., 2018).

"Tejocote" es el nombre con el que se conoce comúnmente a las especies de Crataegus en México. Crataegus mexicana se considera como una de las más importantes a nivel comercial (Betancourt-Olvera, Nieto Ángel, Urbano \& GonzálezAndrés, 2018; Núñez-Colin et al., 2008), aun así, es poca la información relacionada con la composición y cuantificación de los metabolitos secundarios con actividad antioxidante.

Investigaciones recientes han demostrado que algunos subproductos de procesos agroindustriales contienen compuestos bioactivos, principalmente compuestos fenólicos, que actúan sinérgicamente como protectores en contra de agentes oxidantes (Pasqualone et al., 2018). Los compuestos fenólicos constituyen uno de los grupos más numerosos y extensamente distribuidos en el reino vegetal (Vermerris \& Nicholson, 2009). Se ha descrito que los compuestos fenólicos como los flavonoides y las procianidinas tienen propiedades antioxidantes, secuestradoras de radicales libres, anti-inflamatorias, vasorelajantes e hipolipidémicas, además de poseer propiedades antimicrobianas (Gundogdu et al., 2014; Salmanian, Sadeghi, Alami \& Ghorbani, 2014). Reyes-Becerril et al. (2019) reportaron que los compuestos fenólicos extraídos de Crataegus mexicana, nanoencapsulados en maltodextrina, lograron aumentar el porcentaje de células fagocíticas, estimularon la producción de especies reactivas de oxígeno y modularon la capacidad antioxidante, al aumentar la actividad de la enzima superóxido dismutasa en leucocitos de peces de la especie Seriola rivoliana.

La extracción de compuestos fenólicos requiere de la selección cuidadosa de los disolventes y de la metodología de extracción apropiada para evitar su degradación(Ongkowijoyo, Luna-Vital \& González, 2018). La extracción asistida por ultrasonido, al considerarse un método eficiente, de bajo costo, amigable con el medio ambiente y de fácil integración a la industria alimentaria y/o farmacéutica, resulta ser la opción más idónea para su extracción. El objetivo de este estudio fue cuantificar e identificar los compuestos bioactivos extraídos de la cáscara, pulpa y semillas del tejocote, y determinar su potencial antioxidante. La hipótesis del estudio fue que el contenido de compuestos bioactivos y la actividad antioxidante dependen de la fracción del fruto que se estudie, así como de la polaridad del disolvente empleado en la extracción.

\section{MATERIALES Y MÉTODOS}

\section{Reactivos}

Los reactivos Folin-Ciocalteu, 2,2-difenil-1-picrilhidracilo (DPPH), amortiguador Tris- $\mathrm{HCl}$, cloruro férrico hexahidratado, 2,4,6-Tris (2-piridil)-s-triazina (TPTZ), catequina, ácido gálico, ácido 6-hidroxi-2,5,7,8-tetrametil carboxílico (Trolox), 4-hidroxi-3-metoxibenzaldehído (vainillina), sulfato de hierro III, amonio (dodecahidratado) y estándares de HPLC se adquirieron de Sigma-Aldrich (EUA). El ácido clorhídrico, n-butanol y el hidróxido de sodio de J.T. Baker (EUA), el carbonato de sodio de Golden Bell (EUA) y el cloruro de aluminio hexahidratado de los Laboratorios Reasol (México). Los disolventes empleados (acetona y etanol) fueron de grado técnico de la marca Meyer (México).

\section{Recolección del material vegetal e identificación taxonómica} La cosecha de los frutos de tejocote se realizó a inicios del mes de noviembre del 2016, en las instalaciones de la Universidad Autónoma Chapingo, municipio de Texcoco de Mora, estado de México (19²9'23"N, 9853'37"O). La recolecta se realizó en este lugar debido a que los ejemplares ubicados en esta zona se consideran fenotipos superiores en cuanto al tamaño del fruto (Nieto \& Borys, 1993). La identificación taxonómica se llevó a cabo en el Herbario Nacional de México (MEXU) del Instituto de Biología de la UNAM.

\section{Acondicionamiento del material vegetal}

Los frutos cosechados se lavaron con agua corriente y desinfectaron con una solución de hipoclorito de sodio al $0.5 \%$. Posteriormente, los frutos se sometieron a tratamiento térmico en agua en ebullición por 2 min y se enfriaron en un baño de agua a $4{ }^{\circ} \mathrm{C}$ para separar el fruto en tres fracciones: cáscara, pulpa y semilla. La pulpa se congeló inmediatamente con nitrógeno líquido y posteriormente se liofilizó (FreeZone, Labconco, USA). La semilla y la cáscara se secaron en una estufa a $45 \pm 4{ }^{\circ} \mathrm{C}$ por $4 \mathrm{~h}$. Una vez secas, las tres fracciones se pulverizaron en un molino (GX4100, Krups, Alemania) y se tamizaron en una malla de 25 mesh. El material se almacenó en bolsas de plástico bajo condiciones de oscuridad y temperatura ambiente de $22^{\circ} \mathrm{C}$.

\section{Obtención de los extractos}

Los extractos se obtuvieron empleando soluciones acidificadas $(\mathrm{HCl} 2 \mathrm{~N})$ de etanol-agua (70:30) y acetona-agua (70:30). Las 
mezclas se sonicaron en un baño ultrasónico (TI-H-5, Elma, Alemania) empleando $25 \mathrm{kHz}$ de frecuencia, durante $30 \mathrm{~min}$. A continuación, los extractos se centrifugaron(5424-R, Eppendorf, Alemania) durante $15 \mathrm{~min}$ a $1500 \mathrm{x} g$ y los sobrenadantes se filtraron y guardaron en recipientes de vidrio. Con los residuos sólidos resultantes se realizó una segunda extracción en las mismas condiciones de operación. La relación sólido-líquido fue de 1:20. Los sobrenadantes de las dos extracciones se mezclaron y concentraron en un rotavapor (RE500, Yamato, Japón) a $45^{\circ} \mathrm{C}$. Los concentrados se secaron por liofilización y se almacenaron en frascos de vidrio ámbar. El rendimiento de extracción se calculó mediante la siguiente ecuación:

Rendimiento de extracción $(\%)=\left[\frac{\text { masa de extracto seco }(\mathrm{g})}{\text { masa de materia vegetal }(\mathrm{g})}\right] * 100$

\section{Cuantificación de fenoles totales}

El contenido de fenoles totales se calculó a partir de la capacidad de reducción del reactivo Folin-Ciocalteau, siguiendo la metodología de Franco-Bañuelos, Contreras-Martínez, Carranza-Téllez \& Carranza-Concha (2017) con modificaciones de Quiroz-Reyes \& Fogliano (2018). Un volumen de $20 \mu \mathrm{L}$ de muestra, de concentración $1 \mathrm{mg} / \mathrm{mL}$, se adicionó a $1.4 \mathrm{~mL}$ de agua destilada, seguido de $100 \mu \mathrm{L}$ del reactivo Folin-Ciocalteau $(2 \mathrm{~N})$. Se dejó reposar en la oscuridad por 3 min a $25{ }^{\circ} \mathrm{C}$. Terminado el tiempo de reposo y bajo agitación vigorosa, se adicionaron $300 \mu \mathrm{L}$ de una solución de carbonato de sodio (20\%) y $180 \mu \mathrm{L}$ de agua destilada. Después de reposar $100 \mathrm{~min}$ en la oscuridad, la absorbancia de la solución resultante se determinó a una longitud de onda de $760 \mathrm{~nm}$, con el uso de un espectrofotómetro UV-Vis (Multiskan Go, Thermo Fisher, Finlandia). Los resultados se expresaron como la media de la masa equivalente de ácido gálico en $\mathrm{mg}$ por $\mathrm{g}$ de extracto seco (mg EAG / g ES).

\section{Contenido de flavonoides totales}

Para determinar el contenido de flavonoides totales se usó el método colorimétrico descrito por Dewanto, Wu, Adom \& Liu (2002). Se tomaron $200 \mu \mathrm{L}$ de extracto $(1 \mathrm{mg} / \mathrm{mL})$ y se mezclaron con $1 \mathrm{~mL}$ de agua destilada y $60 \mu \mathrm{L}$ de solución de $\mathrm{NaNO}_{2}(5 \%)$. Después de $6 \mathrm{~min}$, se agregaron $120 \mu \mathrm{L}$ de una solución de $\mathrm{AlCl}_{3} \cdot 6 \mathrm{H}_{2} \mathrm{O}(10 \%)$ y la muestra se dejó reposar durante $5 \mathrm{~min}$. Posteriormente, se agregaron $400 \mu \mathrm{L}$ de $\mathrm{NaOH}$ y $220 \mu \mathrm{L}$ de agua destilada bajo agitación vigorosa. La absorbancia de la mezcla resultante se determinó a $510 \mathrm{~nm}$. El contenido de flavonoides totales se expresó como la media de masa equivalente de la catequina en $\mathrm{mg}$ por $\mathrm{g}$ de extracto seco (mg EC / g ES).

\section{Contenido de flavan-3-ol}

Se determinó el contenido de flavan-3-ol usando la metodología descrita por Belščak, Komes, Horžić, Ganić \& Karlović (2009), con algunas modificaciones. Para el análisis, se tomaron $200 \mu \mathrm{L}$ del extracto reconstituido previamente en metanol $(1 \mathrm{mg} / \mathrm{mL})$ y se adicionaron a $1.2 \mathrm{~mL}$ de una solución de vainillina (4\%). La mezcla se agitó vigorosamente en un vortex (Velp, Europe) y después de 5 min de reposo se agregaron $600 \mu \mathrm{L}$ de ácido clorhídrico concentrado. La mezcla se mantuvo en un baño de agua fría a $3 \pm 1{ }^{\circ} \mathrm{C}$ durante $15 \mathrm{~min}$, en la oscuridad. Finalmente, la absorbancia se midió a una longitud de onda de $500 \mathrm{~nm}$. Para cada extracto se preparó un blanco en donde la solución de vainillina se reemplazó por metanol. El contenido de flavan-3-ol se expresó como la media de masa equivalente de catequina en $\mathrm{mg}$ por $\mathrm{g}$ de extracto seco (mg EC/g ES).

\section{Cuantificación de proantocianidinas}

El contenido de proantocianidinas se determinó siguiendo el procedimiento descrito por Quiroz- Reyes \& Fogliano (2018). El ensayo se llevó a cabo mezclando $250 \mu \mathrm{L}$ de extracto $(1 \mathrm{mg} / \mathrm{mL})$ con $1.5 \mathrm{~mL}$ de una solución acidificada de n-BuOH y $50 \mu \mathrm{L}$ de una solución acidificada de $\mathrm{NH}_{4} \mathrm{Fe}\left(\mathrm{SO}_{4}\right)_{2} \cdot 12 \mathrm{H}_{2} \mathrm{O}(2 \%)$. La mezcla se agitó vigorosamente y se calentó en un baño con agua a ebullición durante $45 \mathrm{~min}$. La muestra se enfrió y la absorbancia se registró a una longitud de onda de $550 \mathrm{~nm}$. El valor del blanco (muestra sin calentamiento) se restó y la cantidad de proantocianidinas se determinó a partir de una curva estándar de cloruro de cianidina. El contenido de proantocianidinas se expresó como mg equivalentes de cloruro de cianidina (ECy) por g de extracto seco (mg ECy / g ES).

Evaluación de la capacidad reductora del hierro (FRAP) La actividad antioxidante se evaluó mediante el ensayo de FRAP siguiendo la metodología propuesta por Quiroz-Reyes, Ronquillo-de Jesús, Aguilar-Méndez \& Ramírez-Ortiz (2013). La solución FRAP se preparó mezclando $25 \mathrm{~mL}$ de acetato como amortiguador $(0.3 \mathrm{M}, \mathrm{pH} 3.6), 2.5 \mathrm{~mL}$ de solución TPTZ $(0.01 \mathrm{M})$ y $2.5 \mathrm{~mL}$ de una solución de $\mathrm{FeCl}_{3} \cdot 6 \mathrm{H}_{2} \mathrm{O}(0.02 \mathrm{M})$. La solución FRAP se llevó a una temperatura de $37^{\circ} \mathrm{C}$ previo a su uso. Se tomaron $100 \mu \mathrm{L}$ de muestra $(0.1 \mathrm{mg} / \mathrm{mL})$ y se mezclaron con $1.9 \mathrm{~mL}$ de solución FRAP. La mezcla se agitó vigorosamente y se dejó reposar por $30 \mathrm{~min}$ en la oscuridad. Posteriormente, la absorbancia se registró a $593 \mathrm{~nm}$. Los resultados se reportaron como la media de $\mu \mathrm{M}$ equivalentes de Trolox por $100 \mathrm{ppm}$ de solución de extracto ( $\mu \mathrm{M} \mathrm{ET} \mathrm{/} 100$ ppm SE).

\section{Capacidad secuestradora del radical DPPH}

La capacidad antiradical se determinó sobre el radical DPPH (Brand-Williams, Cuvelier \& Berset, 1995), siguiendo las recomendaciones sugeridas por Molyneux (2004). Una alícuota de $500 \mu \mathrm{L}$ de muestra se mezcló con $125 \mu \mathrm{L}$ de Tris- $\mathrm{HCl}(\mathrm{pH}$ 7.4, $0.1 \mathrm{M})$. A esta mezcla, se le agregaron $500 \mu \mathrm{L}$ de DPPH $(431.1 \mu \mathrm{M})$. Después de $30 \mathrm{~min}$ de reposo en la oscuridad, la absorbancia se registró a $517 \mathrm{~nm}$. Se calculó el $\mathrm{EC}_{50}$, definido como la cantidad de antioxidante necesaria para disminuir en un $50 \%$ la concentración inicial del radical DPPH. Los resultados se expresaron en ppm. El porcentaje de reducción de DPPH se calculó usando la siguiente ecuación: 
Inhibición de DPPH $(\%)=\left[1-\frac{\text { Absorbancia muestra }}{\text { Absorbancia blanco }}\right] * 100$

\section{Análisis de HPLC}

Para cuantificar e identificar los compuestos fenólicos y el ácido ascórbico, se utilizó un cromatógrafo de HPLC serie Agilent 1200 (Agilent Technologies, Waldbronn, Alemania) con un UV-DAD equipado con una columna Zorbax Eclipse Plus C18 (100 x 4.6 mm, $3.5 \mu \mathrm{m}$ ) (Agilent Technologies, Waldbronn, Alemania) siguiendo la metodología propuesta por Quiroz-Reyes et al. (2013) con algunas modificaciones. El termostato del compartimento de la columna se ajustó a $30{ }^{\circ} \mathrm{C}$, el flujo implementado fue de $0.7 \mathrm{~mL} / \mathrm{min}$ y el volumen de inyección de las muestras y los estándares fue de $5 \mu \mathrm{L}$. La elución en gradiente se llevó a cabo empleando agua/ácido fórmico (99.9/0.1), como eluyente A, y metanol/acetonitrilo (50/50), como eluyente B. El gradiente aplicado se realizó de la siguiente manera: $10-15 \%$ B de 0 a $10 \mathrm{~min}, 15-20 \% \mathrm{~B}$ de 10 a $20 \mathrm{~min}$ y $20-32 \%$ B de 20 a 35 min. Previamente, los extractos secos de las muestras se reconstituyeron en metanol y agua desionizada (9/1), y se filtraron a través de un filtro PTFE (Millipore, EUA) con un tamaño de poro de $0.22 \mu \mathrm{m}$; posteriormente, se inyectaron al HPLC-DAD. Se identificaron los compuestos en los extractos comparando los tiempos de retención de las muestras con respecto a los estándares. Los cromatogramas obtenidos se registraron a una longitud de onda de 270 nm utilizando una curva de calibración de los estándares (ácido ascórbico, ácido clorogénico, catequina, epicatequina, procianidina B2, vitexina, quercetina y 3-D-galactósido) para el análisis cuantitativo.

\section{Análisis estadístico}

Se estableció un diseño experimental de bloques completamente aleatorizado considerando dos tratamientos: mezcla acetonaagua (Extracto A) y mezcla etanol-agua (Extracto E), y tres réplicas. Los resultados se analizaron con ANOVA. Diferencias entre las medias de los tratamientos se detectaron mediante
Tukey $(\alpha=0.05)$. Un análisis de regresión lineal simple fue empleado para evaluar la correlación entre FRAP y DPPH $v s$ la concentración de metabolitos secundarios.

\section{RESULTADOS Y DISCUSIÓN \\ Rendimiento de extracción}

La mezcla acetona-agua favoreció significativamente el rendimiento de extracción en las fracciones cáscara y semilla, mientras que en la pulpa el rendimiento fue mayor al utilizar la mezcla etanol-agua $(\mathrm{P}<0.05)$ (Tabla I). Las diferencias en el rendimiento de extracción se deben principalmente al efecto sinérgico entre la metodología de extracción, las propiedades físicas de los disolventes, la afinidad de los metabolitos con los disolventes y el tipo de material vegetal (Miao et al., 2016). La pulpa compuesta en su mayoría por monosacáridos, disacáridos, ácidos orgánicos y pectinas (Franco-Mora, Aguirre-Ortega, Morales-Rosales, González-Huerta \& Gutiérrez-Rodríguez, 2010; Edwards et al., 2012; Lozano-Grande et al., 2016), obtuvo el mayor rendimiento de extracción al emplear la mezcla etanol-agua, debido a que estos compuestos presentan una mayor afinidad por los disolventes polares.

\section{Contenido de fenoles totales y flavonoides totales}

Los polifenoles y flavonoides son de los principales compuestos bioactivos presentes en los frutos del género Crataegus (Miao et al., 2016). La Tabla I muestra el contenido de fenoles totales de las tres fracciones del fruto de tejocote empleando dos mezclas de disolventes de extracción. Se observó que el extracto de la semilla presentó los mayores valores seguido por la cáscara y la pulpa. A su vez, los extractos A de la semilla y la cáscara presentaron contenidos fenólicos significativamente mayores que los extractos $\mathrm{E}(\mathrm{P}<0.05)$. Yilmaz \& Toledo (2006) reportaron que al emplear una mezcla acuosa de acetona, obtuvieron un valor mayor de contenido fenólico en los extractos de la semilla de la uva Muscadina, en comparación con los extractos obtenidos con mezclas acuosas de etanol y metanol. De acuerdo con Koffi, Sea, Dodehe \& Soro (2010), diversos factores influyen en la extracción de los compuestos

Tabla I. Rendimiento de extracción, contenido de fenoles totales, flavonoides totales, flavan-3-ol y proantocianidinas en la cáscara, pulpa y semilla del fruto de tejocote.

\begin{tabular}{|l|c|c|c|c|c|c|}
\hline \multirow{2}{*}{} & \multicolumn{2}{|c|}{ Cáscara } & \multicolumn{2}{c|}{ Pulpa } & \multicolumn{2}{c|}{ Semilla } \\
\cline { 2 - 7 } & Extracto A & Extracto E & Extracto A & Extracto E & Extracto A & Extracto E \\
\hline Rendimiento (\%) & $21.1 \pm 1.4^{\mathrm{a}}$ & $10.7 \pm 0.9^{\mathrm{b}}$ & $40.3 \pm 2.0^{\mathrm{b}}$ & $55.8 \pm 3.1^{\mathrm{a}}$ & $1.7 \pm 0.1^{\mathrm{a}}$ & $1.3 \pm 0.1^{\mathrm{b}}$ \\
\hline CFT (mg EAG / g ES) & $149.8 \pm 3.1^{\mathrm{a}}$ & $78.4 \pm 6.4^{\mathrm{b}}$ & $88.1 \pm 3.5^{\mathrm{a}}$ & $84.8 \pm 3.0^{\mathrm{a}}$ & $251.9 \pm 6.6^{\mathrm{a}}$ & $210.8 \pm 8.4^{\mathrm{b}}$ \\
\hline CFL (mg EC / g ES) & $90.4 \pm 1.2^{\mathrm{a}}$ & $36.1 \pm 2.1^{\mathrm{b}}$ & $40.7 \pm 0.5^{\mathrm{a}}$ & $29.5 \pm 2.8^{\mathrm{b}}$ & $115.6 \pm 3.2^{\mathrm{a}}$ & $55.0 \pm 1.5^{\mathrm{b}}$ \\
\hline FLN (mg EC / g ES) & $60.3 \pm 3.2^{\mathrm{a}}$ & $26.7 \pm 2.1^{\mathrm{b}}$ & $21.8 \pm 2.6^{\mathrm{a}}$ & $22.4 \pm 3.9^{\mathrm{a}}$ & $6.8 \pm 1.1^{\mathrm{a}}$ & $2.3 \pm 0.5^{\mathrm{b}}$ \\
\hline TAC (mg ECy / g ES) & $25.1 \pm 0.4^{\mathrm{a}}$ & $17.4 \pm 0.6^{\mathrm{b}}$ & $12.9 \pm 0.9^{\mathrm{a}}$ & $11.3 \pm 0.2^{\mathrm{a}}$ & $17.9 \pm 0.6^{\mathrm{a}}$ & $15.8 \pm 0.5^{\mathrm{b}}$ \\
\hline $\begin{array}{l}\text { CFT=Contenido de fenoles totales; CFL=Contenido deflavonoides totales; FLN=Contenido de flavan-3-ol; TAC }=\text { Contenidodeproantocianidinas } \\
\text { (taninos condensados). Tratamientos: Extracto A }=\text { Acetona-agua; Extracto E }=\text { Etanol-agua. Valores } \pm \text { sd (n }=3 \text { ). Letras diferentes indican } \\
\text { diferencias significativas entre los tratamientos (P }<0.05) .\end{array}$ \\
\hline
\end{tabular}


fenólicos, como la naturaleza química del material, el método de extracción, el tamaño de la muestra y las condiciones de almacenamiento, entre otros.

En las especies del género Crataegus, los flavonoides son el grupo de compuestos fenólicos más importantes y los responsables de su actividad farmacológica (Refaat et al., 2010). En la Tabla I se puede observar que el contenido de flavonoides totales del extracto de la semilla fue significativamente mayor $(\mathrm{P}<0.05)$ que el contenido de flavonoides de la cáscara y la pulpa. Los resultados del contenido de flavonoides totales muestran que la mezcla de acetona-agua también favoreció la extracción de este tipo de compuestos. Los resultados obtenidos en el presente trabajo difieren a lo señalado por Franco et al. (2008), quienes indican que los mayores rendimientos de compuestos fenólicos se obtienen empleando etanol, metanol y sus mezclas con agua como disolventes de extracción, sin embargo, también señalan que la solubilidad de los polifenoles depende principalmente de los grupos hidroxilo, el tamaño molecular y la longitud de la cadena hidrocarbonada. Por otro lado, Miao et al. (2016) documentaron que el empleo de agua y acetona como disolventes de extracción mejora el rendimiento de los flavonoides totales y otros compuestos fenólicos en comparación con el uso del etanol y metanol.

\section{Contenido de flavan-3-ol y proantocianidinas}

La cáscara presentó el mayor contenido de flavan-3-ol en el extracto A(Tabla I). Este valor fue significativamente mayor (P $<0.05$ ) que los valores obtenidos en los extractos de la pulpa y la semilla. De igual manera, el extracto de la cáscara presentó la mayor concentración de proantocianidinas (Tabla I) cuando se utilizó la mezcla de acetona-agua durante la extracción. Como se sabe, los flavonoles y proantocianidinas pertenecen al grupo más abundante de flavonoides que se pueden encontrar en el género Crataegus, sin embargo, el perfil de las unidades monoméricas y oligoméricas que lo componen se determinan principalmente por la especie de la que se trata y la parte del fruto que ha sido seleccionada para su estudio (Yang \& Liu, 2012).

En la presente investigación, las diferencias en el rendimiento de extracción de los flavonoides dependieron directamente del disolvente de extracción utilizado. Según Galili \& Hovav (2014), la eficiencia de extracción de los compuestos fenólicos se determina por la interacción que existe entre la matriz vegetal y los solutos, o la ruta de difusión de los solutos a través de la matriz vegetal. Esto explica que los extractos A recaudaran el mayor contenido de polifenoles apolares (proantocianidinas) en comparación con los extractos $\mathrm{E}$, ya que estos metabolitos altamente polimerizados y de mayor peso molecular tienen un comportamiento menos polar, y presentan además una fuerte interacción con la pared celular, proteínas, minerales y otras macromoléculas de la matriz vegetal a través de puentes de hidrógeno y enlaces covalentes (Velickovic, Ilic, Mitic, Mitic \& Kostic, 2016); por lo que, las mezclas de disolventes menos polares (i.e. acetona) extraen más flavonoides apolares (i.e. proantocianinas) (Pérez-Jiménez, Díaz-Rubio \& Saura-Calixto 2014).

La síntesis de los flavonoides se promueve por la luz solar, su mayor concentración se ubica en los tejidos exteriores de las plantas (i.e. hojas y cáscaras) (Martínez-Flórez, GonzálezGallego, Culebras \& Tuñón, 2002), lo que explica por qué la concentración mayor de flavan-3-ol y taninos condensados se presentó en la cáscara. Esto también concordó con la concentración mayor de proantocianidinas en la cáscara de manzana (Malus domestica), en contraste con su semillay pulpa (Vázquez-Flores, Álvarez-Parrilla, López-Díaz, Wall-Medrano \& de la Rosa, 2012). Sin embargo, nuestros resultados son contradictorios al compararse con los datos obtenidos para el contenido de flavonoides totales, ya que la semilla resultó obtener la mayor concentración de estos metabolitos. Probablemente, esto se debe a una mayor presencia de flavonoides glucosilados, entre otros subgrupos de flavonoides en el extracto de la semilla que no pertenecen al subgrupo de taninos condensados ni flavan-3-ol.

\section{Análisis de HPLC}

La concentración de los compuestos fenólicos y elácido ascórbico en los extractos de la cáscara, pulpa y semilla del fruto de tejocote se muestran en la Tabla II. Las Figuras 1-3, presentan los cromatogramas de los extractos acuoso-acetónicos (A) y acuosoetanólicos (E) de las tres fracciones del fruto mencionadas, mediante los cuales se identificaron los compuestos: ácido ascórbico, ácido clorogénico, catequina, vitexina, procianidina B2, epicatequina y quercetina-3-D-galactósido (hiperósido).

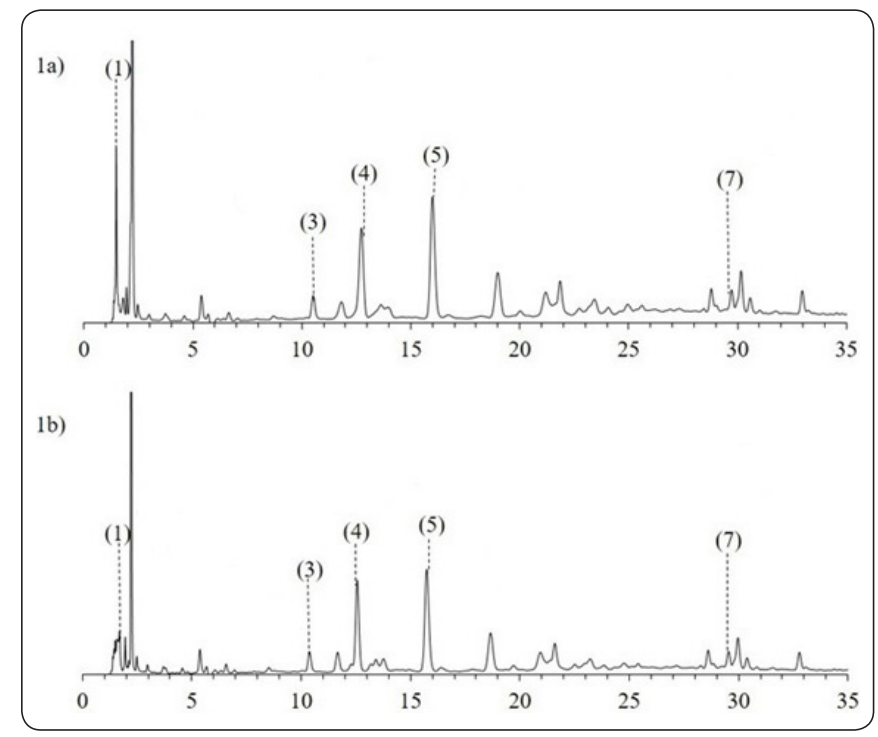

Figura 1. Identificación de compuestos bioactivos por HPLC presentes en la cáscara del tejocote: a) extracto acetona-agua y b) extracto etanol-agua. (1) Ácido ascórbico; (3) ácido clorogénico; (4) procianidina B2; (5) epicatequina; (7) hiperósido. 
Tabla II. Compuestos fenólicos identificados en la cáscara, pulpa y semilla del fruto de tejocote.

\begin{tabular}{|l|c|c|c|c|c|c|}
\hline \multirow{2}{*}{ Compuesto } & \multicolumn{2}{|c|}{ Cáscara (mg/g) } & \multicolumn{2}{c|}{ Pulpa (mg / g) } & \multicolumn{2}{c|}{ Semilla (mg / g) } \\
\cline { 2 - 7 } & Extracto A & Extracto E & Extracto A & Extracto E & Extracto A & Extracto E \\
\hline Catequina & ND & ND & ND & ND & $5.38 \pm 0.05^{\mathrm{a}}$ & $4.93 \pm 0.15^{\mathrm{a}}$ \\
\hline Epicatequina & $11.40 \pm 0.25^{\mathrm{a}}$ & $7.82 \pm 0.14^{\mathrm{b}}$ & $7.34 \pm 0.01^{\mathrm{a}}$ & $4.82 \pm 0.01^{\mathrm{b}}$ & $19.93 \pm 0.04^{\mathrm{a}}$ & $20.02 \pm 0.49^{\mathrm{a}}$ \\
\hline Procianidina B2 & $3.69 \pm 0.01^{\mathrm{a}}$ & $2.49 \pm 0.01^{\mathrm{b}}$ & $2.00 \pm 0.01^{\mathrm{a}}$ & $1.21 \pm 0.01^{\mathrm{b}}$ & $\mathrm{ND}$ & $\mathrm{ND}$ \\
\hline Hiperósido & $2.24 \pm 0.01^{\mathrm{a}}$ & $1.32 \pm 0.11^{\mathrm{b}}$ & $0.28 \pm 0.01^{\mathrm{a}}$ & $0.18 \pm 0.01^{\mathrm{b}}$ & $0.58 \pm 0.05^{\mathrm{a}}$ & $0.81 \pm 0.14^{\mathrm{a}}$ \\
\hline Vitexina & $\mathrm{ND}$ & $\mathrm{ND}$ & $\mathrm{ND}$ & $\mathrm{ND}$ & $3.39 \pm 0.21^{\mathrm{a}}$ & $2.49 \pm 0.02^{\mathrm{b}}$ \\
\hline Ac. Ascórbico & $25.44 \pm 2.24^{\mathrm{a}}$ & $2.57 \pm 0.06^{\mathrm{b}}$ & $3.48 \pm 0.10^{\mathrm{a}}$ & $1.91 \pm 0.08^{\mathrm{b}}$ & $13.51 \pm 0.07^{\mathrm{a}}$ & $12.80 \pm 1.29^{\mathrm{a}}$ \\
\hline Ac. Clorogénico & $4.08 \pm 0.01^{\mathrm{a}}$ & $3.01 \pm 0.04^{\mathrm{b}}$ & $1.38 \pm 0.01^{\mathrm{a}}$ & $1.18 \pm 0.05^{\mathrm{b}}$ & $\mathrm{ND}$ & $\mathrm{ND}$ \\
\hline $\begin{array}{l}\text { ND }=\text { No determinado; Extracto } \mathrm{A}=\text { Acetona-agua; Extracto } \mathrm{E}=\text { Etanol-agua. Valores } \pm \text { sd }(\mathrm{n}=3) \text {. Letras diferentes indican diferencias significativas } \\
\text { entre los tratamientos }(\mathrm{P}<0.05) .\end{array}$
\end{tabular}

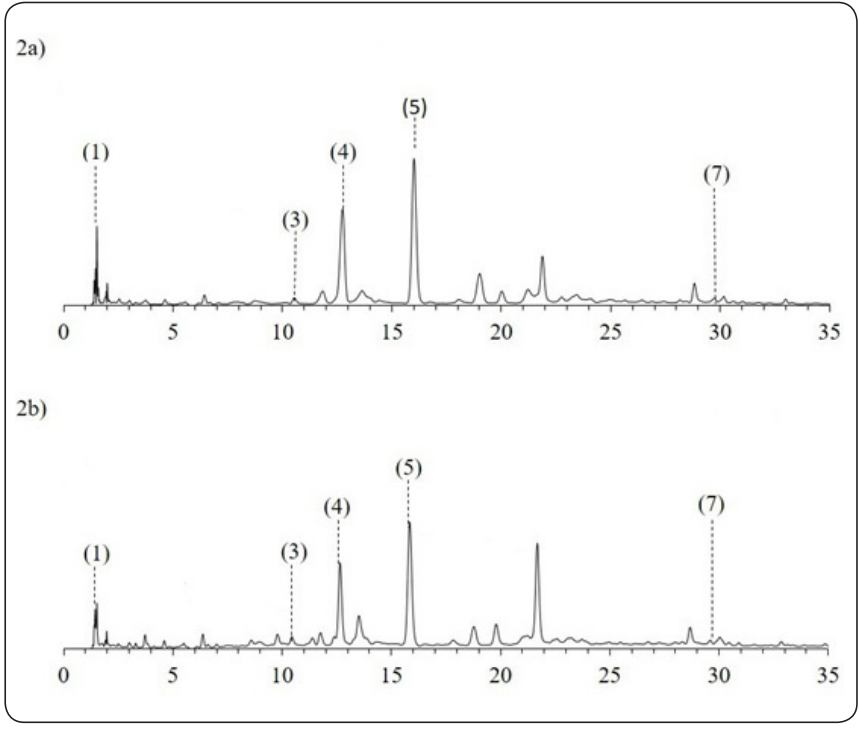

Figura 2. Identificación de compuestos bioactivos por HPLC presentes en la pulpa del tejocote: a) extracto acetona-agua y b) extracto etanol-agua. (1) Ácido ascórbico; (3) ácido clorogénico; (4) procianidina B2; (5) epicatequina; (7) hiperósido.

El ácido ascórbico fue el compuesto que se encontró en mayor concentración en las muestras evaluadas, el extracto A de la cáscara fue el que obtuvo el mayor rendimiento de extracción de este compuesto $(25.44 \pm 2.24 \mathrm{mg} / \mathrm{g})$, con diferencias estadísticamente significativas respecto al extracto $\mathrm{E}$ de la misma fracción $(2.57 \pm 0.06 \mathrm{mg} / \mathrm{g})$. El ácido ascórbico es un potente antioxidante, que actúa protegiendo a la planta de las especies reactivas de oxígeno, por lo que puede encontrarse en mayor concentración en las partes más vulnerables de la planta (i.e. cáscara); probablemente la alta concentración observada en el extracto A de la cáscara haya incrementado su actividad antiradical sin mostrar diferencias estadísticamente

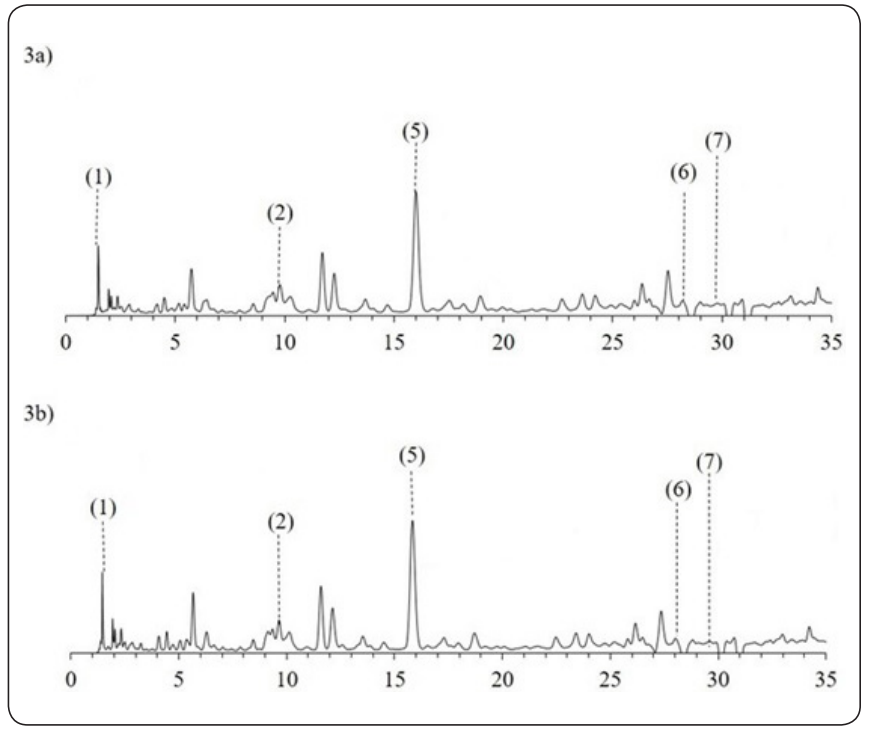

Figura 3. Identificación de compuestos bioactivos por HPLC presentes en la semilla del tejocote: a) extracto acetona-agua y b) extracto etanol-agua. (1) Ácido ascórbico; (2) (+)-catequina; (5) (-)-epicatequina; (6) vitexina; (7) hiperósido.

significativas respecto a la actividad del extracto A de la semilla. Este efecto sinérgico entre compuestos antioxidantes de diferente clase ha sido observado también por otros autores en los extractos del fruto de C. monogyna (Yang \& Liu, 2012; Edwards et al., 2012).

El compuesto quercetina 3-D-galactósido, también conocido como hiperósido, se identificó en los tres tejidos vegetales del fruto de tejocote y tuvo su mayor concentración en la cáscara $(0.14-0.47 \pm 0.01 \mathrm{mg} / \mathrm{g})$. Estos resultados concuerdan con los reportados por Yang \& Liu (2012) en frutos de $C$. pinnatifida (0.1-0.8 mg / g) y Edwards et al. (2012) quienes lo 
identificaron como el mayor flavonol glicosilado cuantificado en frutos de la especie Crataegus. Se ha reportado que diversos factores abióticos tienen un efecto importante en la síntesis de fitoquímicos en frutas y vegetales (Ávila-Sosa, et al., 2017). De acuerdo con Grigelmo-Miguel, Rojas-Grau, Soliva-Fortuny \& Martin-Belloso (2009), la luz solar estimula la síntesis del metabolito quercetina 3-D-galactósido; por lo que suele acumularse principalmente en los tejidos externos (i.e. cáscara y hojas) de frutas y vegetales (Grigelmo-Miguel et al., 2009). Esto explica que el contenido de este compuesto en los extractos de la cáscara fue significativamente mayor que el registrado en los extractos de la pulpa y la semilla.

En la cáscara y la pulpa también se identificaron el ácido clorogénico y la procianidina B2. El ácido clorogénico se identificó como el componente fenólico no flavonoide más abundante en frutos y hojas de la especie de Crataegus, con concentraciones de hasta $12 \mathrm{mg} / \mathrm{g}$. En el presente estudio se obtuvo una concentración máxima de $0.85 \mathrm{mg} / \mathrm{g}$ en el extracto A de la cáscara. Este valor es similar al encontrado por Liu, Kallio, Lu, Zhou \& Yang (2011) en frutos de diferentes cultivos de C. pinnatifida var. major $(0.3-1.6 \mathrm{mg} / \mathrm{g})$ y resultó ser superior a la concentración hallada por Froehlicher et al. (2009) en frutos secos de Crataegus monogyna (0.054 mg / g). La variabilidad entre las concentraciones de éste y otros compuestos fenólicos, se debe principalmente a las diferencias genotípicas entre cada especie; así como al origen, desarrollo y estación en las que se obtuvieron los frutos (Yang \& Liu, 2012; Wyspiańska, Kucharska, Sokół-Łętowska \& KolniakOstek, 2017). El compuesto predominante en los extractos de la pulpa y la semilla fue la epicatequina. El extracto A de la pulpa obtuvo una concentración de epicatequina $(7.34 \mathrm{mg} / \mathrm{g})$ significativamente mayor que la concentración en el extracto $\mathrm{E}$ ( $4.82 \pm 0.01 \mathrm{mg} / \mathrm{g}$ ), sin embargo, no se observaron diferencias significativas entre las concentraciones de epicatequina de los extractos A y E de la semilla. Estos resultados concuerdan con estudios realizados con HPLC-DAD publicados por Miao et al. (2016) del fruto Crataegus spp. en donde una mezcla acetónica $(80 \%)$ obtuvo los mejores rendimientos de este compuesto $(2.710 \pm 0.141 \mathrm{mg} / \mathrm{g})$. Según Oliveira, Mateus \& de Freitas, (2013) la concentración de epicatequina en el fruto de Crataegus se relaciona directamente con el estado de madurez en el que se cosechan los frutos. Durante la maduración del fruto, el contenido de flavan-3-ol (i.e. epicatequina y catequina) y proantocianidinas puede disminuir considerablemente si se compara con la concentración observada en las primeras etapas del desarrollo del fruto (Oliveira et al., 2013).

En los extractos de la semilla de C. mexicana se identificaron los flavonoides catequina y vitexina. La cantidad de vitexina del extracto A de la semilla fue significativamente mayor que la cantidad de vitexina del extracto E (Tabla II). Estos datos corresponden con lo registrado por Yang \& Liu (2012), quienes identificaron a la vitexina y su derivado glicosilado vitexin2"-O-ramanosida como las flavonas con mayor concentración en frutos de tejocote de diversas especies de Crataegus. De acuerdo con Grigelmo-Miguel et al. (2009), la vitexina es un flavonoide de la subclase flavonas cuya presencia en hojas y semillas proporciona protección contra la radiación UV-B. Este tipo de radiación es la que estimula su síntesis y acumulación en el tejido vegetal (Edwards et al., 2012).

Las actividades biológicas documentadas para la especie de Crataegus se asocian con la presencia de este compuesto del que se tienen informes sobre su acción farmacológica como un potente antioxidante, anticancerígeno, antiinflamatorio y eficaz neuroprotector (Malar et al., 2018). Era de esperarse, que los extractos de la semilla en los cuales se identificó este compuesto, presentaran la mayor actividad secuestradora de radicales libres y mayor actividad quelatadora de hierro a diferencia de los extractos de cáscara y pulpa, en los que no se detectó la vitexina.

\section{Capacidad reductora del Hierro (FRAP) y secuestradora del radical DPPH}

En la prueba de capacidad reductora, la presencia de antioxidantes promueve la reducción de hierro oxidado $\left(\mathrm{Fe}^{3+}\right)$ a hierro reducido $\left(\mathrm{Fe}^{2+}\right)$ por donación de electrones (Rabiei, Bekhradnia, Nabavi, Nabavi \& Ebrahimzadeh, 2012). Encontrándose una relación dosis-respuesta en el poder reductor de los extractos del fruto de tejocote. En la Tabla III, se observa que el extracto A de la semilla presentó la mayor capacidad reductora $(\mathrm{P}<0.05)$ en comparación con sus respectivos extractos A de la cáscara y la pulpa.

Tabla III. Capacidad reductora del hierro y secuestradora del radical DPPH de la cáscara, pulpa y semilla del fruto de tejocote.

\begin{tabular}{|l|c|c|c|c|c|c|}
\hline & \multicolumn{2}{|c|}{ Cáscara } & \multicolumn{2}{c|}{ Pulpa } & \multicolumn{2}{c|}{ Semilla } \\
\cline { 2 - 7 } & Extracto A & Extracto E & Extracto A & Extracto E & Extracto A & Extracto E \\
\hline $\begin{array}{l}\text { FRAP } \\
(\mu \mathrm{M} \mathrm{ET} / 100 \text { ppm SE })\end{array}$ & $81.2 \pm 2.1^{\mathrm{a}}$ & $42.2 \pm 2.9^{\mathrm{b}}$ & $39.2 \pm 2.8^{\mathrm{a}}$ & $30.2 \pm 0.9^{\mathrm{b}}$ & $108.7 \pm 3.3^{\mathrm{a}}$ & $75.9 \pm 2.6^{\mathrm{b}}$ \\
\hline $\begin{array}{l}\mathrm{EC}_{50} \\
(\mathrm{ppm} \mathrm{ES})\end{array}$ & $120.2 \pm 5.9^{\mathrm{a}}$ & $196.6 \pm 11.0^{\mathrm{b}}$ & $218.2 \pm 10.0^{\mathrm{a}}$ & $373.7 \pm 15.8^{\mathrm{b}}$ & $98.4 \pm 2.1^{\mathrm{a}}$ & $150.3 \pm 3.1^{\mathrm{b}}$ \\
\hline
\end{tabular}

Tratamientos: Extracto A = Acetona-agua; Extracto $\mathrm{E}=$ Etanol-agua. Valores $\pm \mathrm{sd}(\mathrm{n}=3)$. Letras diferentes indican diferencias significativas entre los tratamientos $(\mathrm{P}<0.05)$. Estándar prueba DPPH: ácido ascórbico $26.8 \pm 0.3 \mathrm{ppm}$. 
Los compuestos fenólicos participan en la estabilización de los radicales libres y metales al actuar como donantes de electrones. Esto explica por qué el extracto de la semilla, presentó el mayor contenido de fenoles, con mejor capacidad reductora de hierro. Regularmente, los compuestos fenólicos actúan deslocalizando uno de sus electrones y lo hacen reaccionar con otros antioxidantes o mediante la unión a metales $(e . g$. $\mathrm{Cu}$ y $\mathrm{Fe}$ ). Sin embargo, el efecto antioxidante mostrado por el extracto depende de su composición y concentración (Soko'1Łetowska, Oszmianski \& Wojdyło, 2007). Se encontraron correlaciones significativas entre la actividad antioxidante (FRAP) vs el contenido fenólico total $(\mathrm{r}=0.93)$, flavonoides totales $(\mathrm{r}=0.95)$, ácido ascórbico $(\mathrm{r}=0.75)$ y ácido clorogénico $(\mathrm{r}=0.88)$.

Con respecto a la capacidad inhibidora del radical DPPH, observamos que el tipo de disolvente tuvo influencia en la actividad antiradical de los extractos. Los extractos E obtuvieron valores de $\mathrm{EC}_{50}$ mayores, esto se traduce en una menor capacidad antiradical, ya que se necesita mayor concentración de estos extractos para inhibir el radical DPPH. Los extractos A de la semilla y la cáscara (Tabla III) fueron los que presentaron mayor actividad antiradical, sin diferencias significativas entre ambos $(\mathrm{P}>0.05)$. Como se sabe, la actividad antioxidante se asocia al contenido de flavonoides, y a la estructura y disposición de los grupos hidroxilo en esos compuestos (Kirakosyan et al., 2003). Los compuestos que poseen una estructura orto 3'4'-hidroxi en el anillo B (e.g. catequinas) y los compuestos que poseen grupos hidroxilo en posición meta en el anillo A (e.g. kaempferol), exhiben una actividad significativamente mayor que los demás compuestos que pertenecen a este grupo (Soko'1-Łe, towska et al., 2007). La actividad antioxidante de los extractos de la semilla y la cáscara en acetona-agua es mayor, porque obtuvieron los $\mathrm{EC}_{50}$ más bajos comparados con los extractos E. Además, estos valores resultaron ser más bajos que los obtenidos por Mraihi et al. (2013) en extractos metanólicos extraídos por ultrasonido de Crataegus azarolus (240 ppm, $\mathrm{EC}_{50}$ semilla; 780 ppm, $\mathrm{EC}_{50}$ cáscara) y Crataegus monogyna (540 ppm, $\mathrm{EC}_{50}$ semilla; 750 ppm, $\mathrm{EC}_{50}$ cáscara). Las diferencias en la capacidad secuestradora de los radicales libres entre estos extractos pueden atribuirse a las características estructurales de los compuestos bioactivos, que determinan la habilidad de estos compuestos de donar electrones (Rabiei et al., 2012).

Es importante mencionar que los perfiles químicos de los flavonoides identificados en los extractos de la cáscara y la semilla fueron distintos. Sin embargo, su actividad antioxidante no presentó diferencias significativas. Esto se puede justificar debido a un efecto sinérgico entre compuestos antioxidantes de diferente clase; por ejemplo: no flavonoides (ácidos fenólicos y ácidos orgánicos) y flavonoides (flavan-3-ol y proantocianidinas). Se observaron correlaciones importantes entre la actividad antiradical (DPPH) vs fenoles totales $(\mathrm{r}=0.70)$, flavonoides totales $(\mathrm{r}=0.78)$, proantocianidinas $(\mathrm{r}=0.73)$, ácido ascórbico $(\mathrm{r}=0.70)$ y ácido clorogénico $(\mathrm{r}=0.83)$.

\section{CONCLUSIONES}

La mezcla de disolvente acetona-agua condujo a obtener los mayores rendimientos de extracción. El contenido de fenoles totales y flavonoides totales fue mayor en los extractos de la semilla de $C$. mexicana, mientras que los contenidos mayores de flavan-3-ol y proantocianidinas se encontraron en la cáscara. La capacidad reductora del hierro se correlacionó directamente con el contenido de los fenoles totales, flavonoides totales, ácido ascórbico y ácido clorogénico. Por otro lado, la actividad secuestradora de los radicales DPPH también se correlacionó directamente con el contenido de fenoles totales, flavonoides totales, proantocianidinas, ácido ascórbico y ácido clorogénico. El fruto de C. mexicana, es una fuente importante de flavonoides y ácidos orgánicos, principalmente, epicatequina, vitexina, ácido ascórbico y ácido clorogénico.

\section{Agradecimientos}

Los autores agradecen a la Secretaría de Investigación y Posgrado del Instituto Politécnico Nacional, el apoyo económico para la realización de este trabajo y especialmente M. Viviana Robles Botero por la beca que le otorgó el Consejo Nacional de Ciencia y Tecnología en su Programa de Becas de Estímulo Institucional de Formación de Investigadores.

\section{REFERENCIAS}

Ávila-Sosa, R., Ávila-Crisóstomo, E., Reyes-Arcos, E. A., CidPérez, T. S., Navarro-Cruz, A. R. \& Ochoa-Velasco, C. E. (2017). Effect of blue and UV-C irradiation on antioxidant compounds during storage of hawthorn (Crataegus mexicana). Scientia Horticulturae, 217, 102-106. https:// doi.org/10.1016/j.scienta.2017.01.016

Belščak, A., Komes, D., Horžić, D., Ganić, K. K. \& Karlović, D. (2009). Comparative study of commercially available cocoa products in terms of their bioactive composition. Food Research International, 42, 707-716. https://doi. org/10.1016/j.foodres.2009.02.018

Betancourt-Olvera, M., Nieto-Ángel, R., Urbano, B. \& González-Andrés, F. (2018). Analysis of the biodiversity of hawthorn (Crataegus spp.) from the morphological, molecular, and ethnobotanical approaches, and implications for genetic resource conservation in scenery of increasing cultivation: the case of Mexico. Genetic Resources and Crop Evolution, 65, 897-916. DOI: 10.1007/s10722-017-0583-4

Brand-Williams, W., Cuvelier, M. E. \& Berset, C. (1995). Use of a free radical method to evaluate antioxidant activity. LWT-Food Science and Technology, 28, 25-30. https://doi. org/10.1016/S0023-6438(95)80008-5

Cervantes-Paz, B., Ornelas-Paz, J. J., Gardea-Béjar, A. A., Yahia, E. M., Ríos-Velasco, C., Zamudio-Flores, P. B., Ruiz-Cruz, S. \& Ibarra-Junquera, V. (2018). Phenolic compounds of hawthorn (Crataegus spp.): Their 
biological activity associated to the protection of human health. [Compuestos fenólicos de tejocote (Crataegus spp.): Su actividad biológica asociada a la protección de la salud humana]. Revista Fitotecnia Mexicana, 41(3), 339-349.

Dewanto, V., Wu, X., Adom, K. K. \& Liu, R. H. (2002). Thermal processing enhances the nutritional value of tomatoes by increasing total antioxidant activity thermal processing enhances the nutritional value of tomatoes by increasing total antioxidant activity. Journal of Agriculture and Food Chemistry, 50, 3010-3014. DOI: 10.1021/jf0115589

Edwards, J. E., Brown, P. N., Talent, N., Dickinson, T. A. \& Shipley, P. R. (2012). A review of the chemistry of the genus Crataegus. Phytochemistry, 79, 5-26. https://doi. org/10.1016/j.phytochem.2012.04.006

Franco, D., Sineiro, J., Rubilar, M., Sánchez, M., Jerez, M., Pinelo, M., Costoya, N. \& Núñez, M. J. (2008). Polyphenols from plant materials: extraction and antioxidant power. Electronic Journal of Environmental, Agricultural and Food, 7, 3210-3216.

Franco-Bañuelos, A., Contreras-Martínez, C. S., CarranzaTéllez, J. \& Carranza-Concha, J. (2017). Total phenolic content and antioxidant capacity of non-native wine grapes grown in Zacatecas, Mexico. Agrociencia, 51, 661-671.

Franco-Mora, O., Aguirre-Ortega, S., Morales-Rosales, E. J., González-Huerta, A. \& Gutiérrez-Rodríguez, F. (2010). Caracterización morfológica y bioquímica de frutos de tejocote (Crataegus mexicana DC) de Lerma y Ocoyoacac, México. Ciencia ergo sum, 17, 61-66.

Froehlicher, T., Hennebelle, T., Martin-Nizard, F., Cleenewerck, P., Hilbert, J.-L., Trotin, F. \& Grec, S. (2009). Phenolic profiles and antioxidative effects of hawthorn cell suspensions, fresh fruits, and medicinal dried parts. Food Chemistry, 115, 897-903. https://doi.org/10.1016/j. foodchem.2009.01.004

Galili, S, \& Hovav, R. (2014). Determination of polyphenols, flavonoids, and antioxidant capacity in dry seeds. En: Polyphenols in Plants. R. R. Watson (ed.), pp: 305-323. Academic Press. London, UK.

García-Mateos, R., Aguilar-Santelises, L., Soto-Hernández, M., Nieto-Ángel, R. \& Kite, G. (2012). Total phenolic compounds, flavonoids and antioxidant activity in the flowers of Crataegus spp. from México. Agrociencia, 46, 651-662.

Grigelmo-Miguel, N., Rojas-Grau, M. A., Soliva-Fortuny, R. \& Martin-Belloso, O. (2009). Methods of analysis of antioxidant capacity of phytochemicals. En: Fruit and Vegetable Phytochemicals: Chemistry, Nutritional Value, and Stability. L. A. de la Rosa, E. Álvarez-Parrilla \& G. A. González-Aguilar (eds.), pp: 271-307. Wiley-Blackwell, USA.

Gundogdu, M., Ozrenk, K., Ercisli, S., Kan, T., Kodad, O. \& Hegedus, A. (2014). Organic acids, sugars, vitamin C content and some pomological characteristics of eleven hawthorn species (Crataegus spp.) from Turkey. Biological Research, 47, 1-5. http://dx.doi.org/10.1186/0717-6287-47-21

Kirakosyan, A., Seymour, E., Kaufman, P. B., Warber, S., Bolling, S. \& Chang, S. C. (2003). Antioxidant capacity of polyphenolic extracts from leaves of Crataegus laevigata and Crataegus monogyna (hawthorn) subjected to drought and cold stress. Journal of Agriculture and Food Chemistry, 51, 3973-3976. https://doi.org/10.1021/jf030096r

Koffi, E., Sea, T., Dodehe, Y. \& Soro S. (2010). Effect of solvent type on extraction of polyphenols from twenty three Ivorian plants. Journal of Animal and Plant Sciences, 5, 550-558.

Liu P, Kallio, H., Lu, D., Zhou, C. \& Yang, B. (2011). Quantitative analysis of phenolic compounds in Chinese hawthorn (Crataegus spp.) fruits by high performance liquid chromatography- electrospray ionisation mass spectrometry. Food Chemistry, 127, 1370-1377. https:// doi.org/10.1016/j.foodchem.2011.01.103

Lozano-Grande, M. A., Valle-Guadarrama, S., AguirreMandujano, E.,Lobato-Calleros, C. S. O.\& Huelitl-Palacios, F. (2016). Films based on hawthorn (Crataegus spp.) fruit pectin and candelilla wax emulsions: characterization and application on Pleurotus ostreatus. Agrociencia, 50, 849-866.

Malar, D. S., Suryanarayanan, V., Prasanth, M. I., Singh, S. K., Balamurugan, K. \& Devi, K. P. (2018). Vitexin inhibits A $\beta$ 25-35 induced toxicity in Neuro-2a cells by augmenting Nrf2/HO-1 dependent antioxidant pathway and regulating lipid homeostasis by the activation of LXR- $\alpha$. Toxicology in Vitro, 50, 160-171. https://doi.org/10.1016/j.tiv.2018.03.003

Martínez-Flórez, S., González-Gallego, J., Culebras, J. \& Tuñón, J. (2002). Los flavonoides: propiedades y acciones antioxidantes. Nutrición Hospitalaria, 17, 271-278.

Miao, J., Li, X., Fan, Y., Zhao, C., Mao, X., Chen, X., Huang, H. \& Gao, W. (2016). Effect of different solvents on the chemical composition, antioxidant activity and alphaglucosidase inhibitory activity of hawthorn extracts. International Journal of Food Science \& Technology, 51, 1244-1251. https://doi.org/10.1111/ijfs.13076

Molyneux, P. (2004). The use of the stable free radical diphenylpicryl-hydrazyl (DPPH) for estimating antioxidant activity. Songklanakarin Journal of Science and Technology, 26, 211-219.

Mraihi, F., Journi, M., Chérif, J. K., Sokmen, M., Sokmen, A. \& Trabelsi-Ayadi, M. (2013). Phenolic contents and antioxidant potential of Crataegus fruits grown in Tunisia as determined by DPPH, FRAP, and $\beta$-carotene/linoleic acid assay. Journal of Chemistry, Article ID 378264, 6 pp.

Nieto, Á. \& Borys, M. W. (1993). El tejocote (Crataegus spp.); un potencial frutícola para la producción de las zonas templadas y frías. Fruticultura Profesional, 54, 64-71.

Núñez-Colín, C. A., Nieto-Ángel, R., Barrientos-Priego, A. F., Segura, S., Sahagún-Castellanos, J. \& Gonzáles-Andrés, F. (2008). Distribución y caracterización eco-climática del género Crataegus L. (Rosaceae, SUBFAM. Maloideae) en 
México. Revista Chapingo Serie Horticultura, 14, 177-184. DOI: $10.5154 /$ r.rchsh.2006.06.027

Oliveira, J., Mateus, N. \& de Freitas V. (2013). Flavanols: catechins and proanthocyanidins. En: Natural Products. K. G Ramawat. \& J.-M. Mérillon (eds.), pp. 1753-1801. Springer. Berlin, Germany.

Ongkowijoyo, P., Luna-Vital, D. A. \& Gonzalez, E. (2018). Extraction techniques and analysis of anthocyanins from food sources by mass spectrometry: An update. Food Chemistry, 250, 113-126. https://doi.org/10.1016/j. foodchem.2018.01.055

Pasqualone, A., Laddomada, B., Spina, A., Todaro, A., Guzmán, C., Summo, C., Mita, G. \& Giannone, V. (2018). Almond by-products: Extraction and characterization of phenolic compounds and evaluation of their potential use in composite dough with wheat flour. LWT-Food Science and Technology, 89, 299-306. https://doi.org/10.1016/j. lwt.2017.10.066

Pérez-Jiménez, J., Díaz-Rubio, M. E. \& Saura-Calixto, F. (2014). Non-extractable polyphenols in plant foods: nature, isolation, and analysis. En: Polyphenols in Plants. R. R. Watson (ed.), pp: 203-218. Academic Press. London, UK.

Phipps, J. B. (1997). Monograph of northern Mexican Crataegus (Rosaceae, subfam. Maloideae). Botanical Research Institute of Texas, USA.

Quiroz-Reyes, C. N. \& Fogliano, V. (2018). Design cocoa processing towards healthy cocoa products: The role of phenolics and melanoidins. Journal of Functional Foods, 45, 480-490. https://doi.org/10.1016/j.jff.2018.04.031

Quiroz-Reyes, C. N., Ronquillo-de Jesús, E., Aguilar-Méndez, M. A. \& Ramírez-Ortíz, M. E. (2013). Comparative study of ultrasound and maceration techniques for the extraction of polyphenols from cocoa beans (Theobroma cacao L.). Revista Mexicana de Ingeniería Quimica, 12, 11-18.

Rabiei, K., Bekhradnia, S., Nabavi, S. M., Nabavi, S. F, \& Ebrahimzadeh, M. A. (2012). Antioxidant activity of polyphenol and ultrasonic extracts from fruits of Crataegus pentagyna subsp. elburensis. Natural Product Research, 26, 2353-2357. https://doi.org/10.1080/14786419.2012 .658799

Refaat,A. T., Shahat, A.A., Ehsan, N.A., Yassin, N., Hammouda, F., Tabl, E. A. \& Ismail, S. I. (2010). Phytochemical and biological activities of Crataegus sinaica growing in Egypt. Asian Pacific Journal of Tropical Medicine, 3, 257-261. https:/doi.org/10.1016/S1995-7645(10)60062-4
Reyes-Becerril, M., Martínez-Preciado,A., Guluarte, C., Guerra, K., Tovar-Ramírez, D., Macías, M. E. \& Angulo, C. (2019). Phytochemical composition and immunobiological activity of hawthorn Crataegus mexicana nanoencapsulated in longfin yellowtail seriola rivoliana leukocytes. Fish and Shellfish Immunology, 92, 308-314. DOI: 10.1016/j. fsi.2019.06.024

Rigelsky, J. M. \& Sweet, B. V. (2002). Hawthorn: pharmacology and therapeutic uses. American Journal of HealthSystem Pharmacy, 59, 417-422. https://doi.org/10.1093/ ajhp/59.5.417

Salmanian, S., Sadeghi,A. R., Alami, M. \& Ghorbani, M. (2014). Phenolic content, antiradical, antioxidant, and antibacterial properties of Hawthorn (Crataegus elburensis) seed and pulp. Journal of Agricultural Science and Technology, 16, 343-354.

Soko'ł-Łetowska, A., Oszmianski, J. \& Wojdyło, A. (2007). Antioxidant activity of the phenolic compounds of hawthorn, pine and skullcap. Food Chemistry, 103, 853-859. https://doi.org/10.1016/j.foodchem.2006.09.036

Vázquez-Flores, A. A., Álvarez-Parrilla, E., López-Díaz, J. A., Wall-Medrano, A. \& de la Rosa L. A. (2012). Taninos hidrolizables y condensados: naturaleza química, ventajas y desventajas de su consumo. Tecnociencia, VI, 84-93.

Velickovic, J. M., Ilic, S., Mitic, S. S., Mitic, M. N. \& Kostic, D.A. (2016). Comparative analysis of phenolic and mineral composition of hawthorn and blackthorn from southeast Serbia. Oxidation Communications, 39, 2280-2290.

Vermerris, W. \& Nicholson, R. (2009). Phenolic Compound Biochemistry. Springer. The Netherlands.

Wyspiańska, D., Kucharska, A. Z., Sokół-Łętowska, A. \& Kolniak-Ostek, J. (2017). Physico-chemical, antioxidant, and anti-inflammatory properties and stability of hawthorn (Crataegus monogyna Jacq.) procyanidins microcapsules with inulin and maltodextrin. Journal of the Science of Food and Agriculture, 97, 669-678. DOI: 10.1002/jsfa.7787

Yang, B. \& Liu P. (2012). Composition and health effects of phenolic compounds in hawthorn (Crataegus spp.) of different origins. Journal of the Science of Food and Agriculture, 92, 1578-1590. DOI: 10.1002/jsfa.5671

Yilmaz, Y. \& Toledo, R. T. (2006). Oxygen radical absorbance capacities of grape/wine industry byproducts and effect of solvent type on extraction of grape seed polyphenols. Journal of Food Composition and Analysis, 19, 41-48. https://doi.org/10.1016/j.jfca.2004.10.009 